\title{
The cost of long term therapy for gastro-oesophageal reflux disease: a randomised trial comparing omeprazole and open antireflux surgery
}

H E Myrvold, L Lundell, P Miettinen, S A Pedersen, B Liedman, J Hatlebakk, R Julkunen, K Levander, M Lamm, C Mattson, J Carlsson, N O Ståhlhammar, the Nordic GORD Study Group

Trondheim University Hospital, Norway H E Myrvold

Department of Surgery, Sahlgrenska University Hospital, Gothenburg, Sweden L Lundell

B Liedman

University Hospital, Kuopio, Finland P Miettinen

R Julkunen

Odense Hospital, Odense, Denmark S A Pedersen

Haukeland Hospital, Bergen, Norway J Hatlebakk

Ersta Hospital, Stockholm, Sweden K Levander

AstraZeneca R\&D, Mölndal, Sweden M Lamm

C Mattson

J Carlsson

N O Ståhlhammar

Correspondence to: Professor H E Myrvold, Department of Surgery, Regionsykehuset in

Trondheim, N-7006

Trondheim, Norway.

helge.myrvold@

medisin.ntnu.no

Accepted for publication 8 January 2001

\begin{abstract}
Background and aim-To comprehensively assess the relative merits of medical and surgical therapy for gastrooesophageal reflux disease (GORD), health economic aspects have to be incorporated. We have studied the direct and indirect costs of medical and surgical therapy within the framework of a prospective randomised multicentre trial.

Methods-After initial treatment of reflux oesophagitis with omeprazole to control symptoms and to heal oesophagitis, 154 patients were randomised to continue treatment with omeprazole (20 or $40 \mathrm{mg}$ daily) and 144 patients to have an open antireflux operation (ARS). In case of GORD relapse, patients allocated to omeprazole were offered ARS and those initially operated on had either a reoperation or were treated with omeprazole. The costs were assessed over five years from randomisation.

Results-Differences in cumulative direct medical costs per patient between the two therapeutic strategies diminished with time. However, five year direct medical costs per patient when given omeprazole were still significantly lower than for those having ARS in Denmark, Norway, and Sweden (differences were DKK 8703 (US\$1475), NOK 32992 (US\$ 5155), and SEK 13036 (US\$ 1946), respectively). However, in Finland the reverse was true (the difference in favour of ARS amounted to FMK 7354 (US\$ 1599)). When indirect costs (loss of production due to GORD related sick leave) were also included, the cost of surgical treatment increased substantially and exceeded the cost of medical treatment in all countries.

Conclusions-The total costs of medical therapy for chronic GORD were lower than those of open ARS when prospectively assessed over a five year period, although significant differences in cost estimates were revealed between countries.
\end{abstract}

(Gut 2001;49:488-494)

Keywords: proton pump inhibitors; gastro-oesophageal reflux disease; antireflux surgery; omeprazole; oesophagitis; direct medical costs; indirect medical costs
Gastro-oesophageal reflux disease (GORD) is common, therapy of which has developed rapidly in recent years. ${ }^{12}$ In a significant proportion of patients, symptom control and maintained quality of life can only be attained by either continuous proton pump inhibitor (PPI) therapy or antireflux surgery (ARS), both in the short and long term..$^{3-6}$

The relative merits of these therapeutic strategies have been debated in recent years ${ }^{7}$ and to reach a balanced and comprehensive view of the pros and cons of respective therapy, cost aspects have to be introduced. ${ }^{8-12}$ Studies have been presented to show that omeprazole treatment is cheaper than surgery, whereas most investigations have come to conclusions favouring surgery as the most cost effective option. However, these studies have either been retrospective and/or applied a computer based modelling approach. Although valuable information can be obtained from such studies, they all suffer from inherent and well known methodological weaknesses. Furthermore, only exceptionally has a societal perspective been applied, which requires an assessment of both direct and indirect costs.

As an integrated part of a prospective randomised multi-institutional clinical trial comparing omeprazole with open ARS in the long term management of patients with oesophagitis, we have prospectively acquired information on direct as well as indirect costs (that is, costs for loss of production) over a five year period.

\section{Patients and methods}

The study was carried out in Denmark, Finland, Norway, and Sweden and, according to the protocol, comprised a five year follow up period. Patient recruitment started in February 1991 and follow up ended in April 1999.

Eligible for inclusion into the clinical study were patients with chronic reflux symptoms and oesophagitis (of at least grade II according to the Savary-Miller classification) verified at endoscopy performed prior to inclusion. ${ }^{13}{ }^{14}$ These patients were initially given either 20 or $40 \mathrm{mg}$ of omeprazole daily for up to four months to control symptoms and to heal oesophagitis. Successfully treated patients were then randomised to either maintenance

Abbreviations used in this paper: GORD, gastro-oesophageal reflux disease; PPI, proton pump inhibitor; ARS, antireflux surgery; CRF, case report form. 
treatment with omeprazole, using the same dose as needed to heal oesophagitis (20 or 40 mg once daily) or to open ARS. Patients randomised to surgery could be given omeprazole until the time of operation which should, according to the protocol, have been performed within three months of randomisation (actual median interval was 71 days). Details of surgery have been reported previously. ${ }^{13}$

Patients whose oesophagitis did not heal or who had incomplete symptom control at the preoperative endoscopy $(n=34)$ were treated surgically and followed according to the protocol, but otherwise these patients did not participate in the health economic analysis. This means that the economic analysis, as well as the clinical study itself, was set up to compare continuous medical treatment with surgery in patients initially treated successfully with omeprazole.

The protocol stated that each patient should have a hospital based follow up visit every six months for scoring of symptoms and registration of eventual adverse events. After three and nine months of therapy, a similar assessment was done by telephone.

Endoscopic examinations of the oesophagus, stomach, and duodenum were performed within nine days prior to inclusion to verify that oesophagitis was healed. Endoscopic examinations were thereafter performed at the 12,36 , and 60 month visits or at the time of an eventual relapse of symptoms.

In patients allocated to omeprazole, a dose adjustment was allowed in case of symptom and/or oesophagitis relapse. Of the 155 patients allocated to omeprazole, $139 \mathrm{had} 20 \mathrm{mg}$ and 16 patients $40 \mathrm{mg}$ daily. Only a minority of patients had the dose subsequently adjusted due to symptom relapse. ${ }^{15}{ }^{16}$ Only one patient took the maximally allowed dose of $60 \mathrm{mg}$ daily. Patients not responding to higher daily doses of omeprazole ( $\geqslant 60 \mathrm{mg}$ ) were offered surgical treatment. Likewise, patients who relapsed after surgery could, according to the protocol, either have a reoperation or be prescribed omeprazole whereupon the respective costs of these remedial therapies were recorded.

GENERAL CONSIDERATIONS REGARDING THE COST ANALYSIS

A common problem when utilising clinical trials for any kind of cost assessment arises from the fact that the clinical protocol often mandates more visits, consultations, and examinations than otherwise used in clinical practice. Therefore, in order not to overestimate the costs, measures were taken to exclude protocol driven endoscopies and visits to a doctor. The rules for this exercise are described below.

This study used data from both the case report form (CRF) and a special health economic questionnaire completed by the patients at each visit. The health economic questionnaire was used to obtain data on employment, sick leave, and transportation.

An analysis was done separately for each participating country using trial wide data on resource consumption in non-monetary terms and country specific prices. The use of trial wide data on resource consumption was justified by the similarity of the health care systems in the four participating countries. However, the use of trial wide data on sick leave deserves special attention as there are some differences in the systems of social insurance (for example, the degree of compensation in case of sick leave).

The perspective for assessment of costs was societal. Total costs were however divided into direct medical costs, direct non-medical costs, and indirect costs.

DIRECT MEDICAL COSTS

Direct medical costs considered were the costs of operation, endoscopy, visits to the outpatient clinic, and medication.

The unit costs of an operation, reoperation, incisional hernia surgery, endoscopy, and visits were derived from internal assessments of costs at a defined hospital in each participating country. The cost of an operation included the so-called hotel cost (days in hospital).

While all extra endoscopies were included in the calculations, the protocol driven endoscopies at 12,36 , and 60 months were included only if moderate or severe symptoms suggesting recurrence were registered on that particular occasion.

Furthermore, while the first visit as well as all extra visits were included in the calculations, other protocol driven visits were included only if moderate or severe symptoms were registered. However, in the medical arm protocol driven visits at $12,24,36,48$, and 60 months were included regardless of symptom status as it was assumed that the patient had to visit the doctor once every year in order to get a drug prescription. Correspondingly, yearly prescription refill visits were assumed for patients in the surgical treatment arm who required omeprazole for more than one year. In addition, it was assumed that there was a follow up visit after surgery to ensure wound healing and postoperative rehabilitation (those visits were not registered in the CRF).

Inclusion of the first visit might be questioned as it could be regarded as a sunk cost preceding the choice of therapy. However, its inclusion did not affect the comparison between the two treatment arms.

Visits to a nurse were not registered in the CRF. However, a visit to a nurse was assumed to have occurred whenever a health economic questionnaire had been completed with no corresponding information noted in the CRF.

The basis for the calculation of the cost of medication was the number of capsules consumed in each arm. It was however not possible to include consumption of omeprazole that surgically treated patients had before they were operated on as this was not registered. The impact of a similar omission is assessed in the sensitivity analysis.

TRANSPORTATION

The only direct non-medical cost considered was that of transportation in relation to 
surgery, endoscopy, and visits. Data on mode and travelling time per mode of transportation were collected at each visit. Based on observations during the first three years of the study, mean travel time per mode of transportation for a typical visit was calculated. By assuming a mean travelling speed for a private car, taxi, and bus, respectively, and applying a specific cost per $\mathrm{km}$, this information was transformed into a travelling cost per visit. This cost was applied to all travelling for visits, endoscopies, and surgery, irrespective of the treatment arm.

\section{LOSS OF PRODUCTION DUE TO ABSENCE FROM}

WORK

The only indirect cost considered was the loss of production due to GORD related absence from work. Data on employment, number of days on sick leave due both to GORD and other diseases, and absence from work for any companion to the patient were collected at each visit. The number of days on sick leave were transformed into the number of full days using information about the patient's employment status (that is, two days on sick leave for a patient employed half time were transformed into one full day off work). The number of days on sick leave was calculated per patient employed at the time of inclusion in the study.

Due to the design of the protocol we encountered some problems in collecting complete data on sick leave in relation to surgery. To circumvent this, data on all Swedish patient sick leave in connection with the date of surgery were acquired from the Swedish social insurance database. The number of days on sick leave was found to be 32 per employed patient, a value which was used for all employed patients during the first six months of the postoperative recovery period.

For a patient not on sick leave, a visit to a doctor was estimated to require two hours of absence from work and six hours for an endoscopy.

PRICES/UNIT COSTS

All prices/unit costs used in the calculations are presented in table 1. All costs refer to 1996 prices. Prices for medication equalled the retail price. Prices of other direct medical cost items came from estimates provided by participating clinics.

The estimated unit costs of transportation were obtained from bus and taxi companies.

The cost of one hour of absence from work was set equal to the total labour cost per hour for an industrial worker.

\section{STATISTICAL ANALYSIS}

Statistical analysis applied an intention to treat approach. Patients lost to follow up were assumed to incur no costs in the base case analysis. However, the effect of this assumption was assessed in a sensitivity analysis.

For reasons mentioned above, information on transportation and sick leave were regarded as less reliable than information on direct medical costs. Therefore, only descriptive statistics were used for transportation and sick leave estimates. For the difference in direct medical costs, confidence intervals were calculated using the Student's $t$ distribution.

Because the study covered more than one year, costs occurring after the first year were discounted by an annual rate of $3 \%$. The immediate costs associated with all patients recruited into the surgical arm have previously been presented. ${ }^{15}$

\section{SENSITIVITY ANALYSIS}

The sensitivity analysis was limited to direct medical costs. Firstly, two extreme scenarios, relating to unit costs, were constructed in order to assess the robustness of the results with regard to uncertainties in the cost estimates. In one scenario, favouring the medical treatment arm, the costs of visits were arbitrarily reduced by $20 \%$ and that of an endoscopy, operation, reoperation, and incisional hernia surgery increased by $20 \%$. In addition, the rate of discount was increased to $5 \%$ which also favours medical treatment as it reduces the burden of future costs. In the scenario favouring surgical therapy, corresponding costs were changed in the opposite direction. The discount rate in this scenario was reduced to $1 \%$. Secondly, an extrapolation was done for each patient who was lost to follow up. The cost per day while in

Table 1 Prices/unit costs in 1996 prices, local currencies. Rates of exchange to USD are from November 1996

\begin{tabular}{|c|c|c|c|c|}
\hline & $\begin{array}{l}\text { Denmark }^{1} \\
\text { USD } 1=\text { DKK } 5.9\end{array}$ & $\begin{array}{l}\text { Finland }^{2} \\
\text { USD } 1=F M K 4.6\end{array}$ & $\begin{array}{l}\text { Norway }^{3} \\
\text { USD } 1=\text { NOK } 6.4\end{array}$ & $\begin{array}{l}\text { Sweden }{ }^{4} \\
\text { USD } 1=\text { SEK } 6.7\end{array}$ \\
\hline Surgery (incl. hospital stay) & 34530 & 23000 & 66000 & 41759 \\
\hline Endoscopy & 1050 & 600 & 833 & 1328 \\
\hline Visit to doctor & 475 & 350 & 158 & 460 \\
\hline Visit to nurse & 394 & 200 & 131 & 501 \\
\hline Renumeration & 34530 & 20000 & 66000 & 49515 \\
\hline Hernia operation & 20000 & 11500 & 18260 & 25900 \\
\hline Omeprazole $20 \mathrm{mg}$ capsule & 17.9 & 21.9 & 21.8 & 22.4 \\
\hline Omeprazole $40 \mathrm{mg}$ capsule & 34.4 & 35.7 & 42.6 & 29.5 \\
\hline Car $10 \mathrm{~km}^{5}$ & 23.0 & 18.9 & 30.5 & 27.5 \\
\hline Bus $10 \mathrm{~km}^{6}$ & 9.0 & 12.0 & 15.3 & 12.9 \\
\hline Taxi $10 \mathrm{~km}^{6}$ & 91.0 & 60.5 & 107.0 & 107.5 \\
\hline Loss of production per hour of absence & 139.5 & 58.5 & 117.3 & 167.9 \\
\hline
\end{tabular}

${ }^{1}$ Estimates of direct medical costs are the averages of estimates from Odense University Hospital and Aalborg Hospital.

${ }^{2}$ Estimate of cost of surgery is from Diakonissanstaltens Sjukhus, Helsinki. Estimates of other direct medical costs are from University Hospital of Kuopio.

${ }^{3}$ Estimates of direct medical costs are from Aker Hospital, Oslo.

${ }^{4}$ Estimates of direct medical costs are from Sahlgren's University Hospital, Gothenburg.

${ }^{5}$ Based on remuneration for private car in work.

${ }^{6}$ Based on estimates from bus and taxi companies.

${ }^{7}$ Based on the total labour cost per hour for an industrial worker, official statistics. 
Table 2 Patient description and treatment discontinuation

\begin{tabular}{|c|c|c|}
\hline & $\begin{array}{l}\text { Medical } \\
\text { treatment }\end{array}$ & Surgery \\
\hline Randomised & 155 & 155 \\
\hline \multicolumn{3}{|l|}{ Excluded due to } \\
\hline Oesophagitis not verified & - & 1 \\
\hline No intake of medication & 1 & - \\
\hline Lung cancer & - & 1 \\
\hline Refused surgery & - & 9 \\
\hline Included in the analysis & 154 & 144 \\
\hline \multicolumn{3}{|l|}{ Baseline characteristics } \\
\hline \multicolumn{3}{|l|}{ Sex } \\
\hline Male & 115 & 110 \\
\hline Female & 39 & 34 \\
\hline \multicolumn{3}{|l|}{ Age $(y)$} \\
\hline$<50$ & 60 & 69 \\
\hline $50-64$ & 65 & 55 \\
\hline$>64$ & 29 & 20 \\
\hline \multicolumn{3}{|l|}{ Duration of GORD } \\
\hline $1-5 y$ & 48 & 45 \\
\hline $6-10 y$ & 44 & 43 \\
\hline$>10 \mathrm{y}$ & 62 & 56 \\
\hline Barrett's oesophagus & 26 & 22 \\
\hline Strictures & 9 & 11 \\
\hline \multicolumn{3}{|l|}{ Withdrawn due to } \\
\hline Adverse event & 10 & 3 \\
\hline Non-compliance/refusal & 6 & 12 \\
\hline Lost to follow up/other & 5 & 7 \\
\hline Completed 5 y follow up & 133 & 122 \\
\hline
\end{tabular}

the study was calculated for each patient, excluding the cost of surgery, and was extrapolated up to five years. In a third analysis, consumption of medication while waiting for surgery was accounted for by applying an assumption that all patients took one capsule of omeprazole $20 \mathrm{mg}$ per day during the waiting period. In a final sensitivity analysis the effect of excluding protocol driven costs was assessed by including all visits and endoscopies.

\section{Results}

Initially 155 patients were randomised to ARS but nine refused surgery, one was excluded due to a subsequently diagnosed bronchial carcinoma, and one patient was found to lack verified oesophagitis at the preinclusion endoscopy. A total of 155 patients were randomised to long term medical treatment but one patient who never took any study medication was excluded from the analysis. During the five years, 22 patients were withdrawn from the surgical treatment group and 21 from the medical treatment group. The average time in the study for these patients was 864 days. Reasons for withdrawal are given in table 2 . Patients in the two arms were well matched with regard to baseline characteristics (table 2).

At baseline, 99 patients in the surgical arm and 103 randomised to omeprazole completed the health economic questionnaire. The number of patients who completed the questionnaire at the subsequent visits varied between 60 and 86 among the operated patients and between 72 and 93 among those treated with omeprazole.

The clinical results are presented elsewhere, ${ }^{16}{ }^{17}$ but the main result was that analysis of time to treatment failure revealed a significant difference in favour of surgery $(\mathrm{p}<0.001)$. However, when dose adjustment of omeprazole was allowed to control symptoms, no significant difference was found between the two arms $(p=0.088)$. The two treatments were also found to result in maintained normalisation of quality of life.

Resource consumption in non-monetary terms during years $1-5$ is presented in table 3 . Seven patients in the medical arm had an antireflux operation during the first year, six patients in year 2, and three in year 4 . The mean postoperative hospital stay after ARS was 7.2 days.

The total number of endoscopies was similar in the two treatment groups: 442 in the omeprazole group and 424 in the operated patients. When protocol driven endoscopies were excluded, the respective numbers were reduced to 52 and 69 . Thus the number of endoscopies per patient during the five years was 0.34 in the medical arm and 0.44 in the surgical arm.

The cumulative number of visits to a doctor (excluding endoscopy visits but incorporating follow up visits after surgery) was 1199 in the medical and 1222 in the surgical groups. When the number of visits was calculated according to the rules described in the methods section, the numbers dropped to 864 and 388 , respectively. The corresponding number of visits per patient during the five years was 5.6 on omeprazole and 2.7 after ARS. The number of visits to a nurse was similar in the two treatment groups (114 $v 103)$.

A redo-fundoplication was carried out in five patients due to GORD relapse; one patient during the first year, one during the second, two had a reoperation during the fourth, and one during the fifth year after inclusion. Twenty two additional operations (in 15 patients) were done in the surgical group; six incisional hernias were operated on during the

Table 3 Resource consumption per patient and year $(Y)$ in non-monetary terms

\begin{tabular}{|c|c|c|c|c|c|c|c|c|c|c|}
\hline & \multicolumn{5}{|c|}{ Medical treatment } & \multicolumn{5}{|c|}{ Surgical treatment } \\
\hline & $Y 1$ & $Y 2$ & $Y 3$ & $Y 4$ & Y5 & $Y 1$ & $Y 2$ & $Y 3$ & $Y 4$ & Y5 \\
\hline Operation & 0.05 & 0.04 & 0 & 0.02 & 0 & 1 & 0 & 0 & 0 & 0 \\
\hline Endoscopy & 0.15 & 0.03 & 0.06 & 0.04 & 0.06 & 0.21 & 0.08 & 0.11 & 0.01 & 0.07 \\
\hline Visit to doctor & 2.06 & 0.94 & 0.92 & 0.86 & 0.83 & 2.15 & 0.13 & 0.15 & 0.13 & 0.13 \\
\hline Visit to nurse & 0.68 & 0.02 & 0.01 & 0.01 & 0.01 & 0.68 & 0 & 0.01 & 0 & 0.02 \\
\hline Reoperation & 0 & 0 & 0 & 0 & 0 & 0.01 & 0.01 & 0 & 0.01 & 0.01 \\
\hline Hernia & 0 & 0 & 0 & 0.01 & 0 & 0.04 & 0.05 & 0.03 & 0.02 & 0.01 \\
\hline Ome $20 \mathrm{mg}$, capsules & 280.3 & 247.7 & 237.0 & 224.0 & 231.1 & 3.3 & 19.8 & 28.6 & 32.3 & 59.9 \\
\hline Ome $40 \mathrm{mg}$, capsules & 64.6 & 59.2 & 64.0 & 52.1 & 55.7 & 1.1 & 7.7 & 12.5 & 18.4 & 40.6 \\
\hline Car $(\mathrm{km})$ & 63.5 & 22.0 & 21.5 & 20.3 & 19.5 & 88.4 & 5.6 & 6.8 & 3.8 & 5.2 \\
\hline Bus (km) & 17.1 & 5.9 & 5.8 & 5.5 & 5.2 & 23.7 & 1.5 & 1.8 & 1.0 & 1.4 \\
\hline Taxi $(\mathrm{km})$ & 2.4 & 0.8 & 0.8 & 0.8 & 0.7 & 3.3 & 0.2 & 0.3 & 0.1 & 0.2 \\
\hline Days on sick leave ${ }^{1}$ & 3.5 & 3 & 2.1 & 0.2 & 0.3 & 34.8 & 0.07 & 0.15 & 0 & 0 \\
\hline Companions' absence from work (days) & 0.08 & 0.01 & 0.01 & 0 & 0 & 0.12 & 0.01 & 0.01 & 0 & 0 \\
\hline
\end{tabular}

${ }^{1}$ Per patient employed at baseline. 
first postoperative year, seven during the second, four during the third, three during the fourth, and two during the fifth postoperative year. In the medical arm, one incisional hernia had to be repaired during the fourth year after inclusion.

In the omeprazole treatment arm the average patient consumed 1220 capsules containing 20 mg omeprazole (total 187884 capsules in the whole group) and 296 capsules containing 40 mg of omeprazole (total 45534 capsules in the whole group). Each patient randomised to surgery consumed on average 144 capsules (20 $\mathrm{mg}, 20735$ in total) and 80 capsules $(40 \mathrm{mg}$, 11575 in total). Consumption of omeprazole while waiting for surgery was, as noted above, not measured.

The average transportation time to an outpatient visit or to an endoscopy was calculated to be 55 minutes, based on information from 1494 questionnaires. This time period was subdivided into transportation by private car (37 minutes), by public transportation (14 minutes), by taxi (one minute), and by other modes of transportation (three minutes). Assuming a mean travelling speed of 35, 25, and $45 \mathrm{~km}$ per hour for private car, bus, and taxi, this corresponded to a driving distance of $21.6 \mathrm{~km}, 5.8 \mathrm{~km}$, and $0.8 \mathrm{~km}$, respectively.

GORD related sick leave was calculated per patient employed at baseline. In the surgical arm, 74 of the 99 patients who answered the health economic questionnaire were employed. The corresponding value in the medical treatment group was 93 of 103 . GORD related sick leave differed substantially between the two treatment arms. The average days of sick leave per employed patient amounted to 35 days in the surgical arm. This estimation was based both on information from the Swedish insurance office regarding sick leave in connection with the operation ( 32 days), and on data from the health economic questionnaire regarding the period six months to five years after operation. As expected, substantial sick leave was required in close association with the operation, whereas after operation it decreased considerably. GORD related sick leave in the medical treatment group was only 9.1 days per employed patient.

Companions' absence from work was almost identical in the two study arms (0.11 $v 0.14$ days per patient). costs

The direct medical costs in Denmark, Norway, and Sweden were found to be significantly lower for medical treatment than after surgery. Differences in direct medical costs in Denmark were DKK 8703, in Norway NOK 32 092, and in Sweden SEK 13 036. (Applying rates of exchange from November 1996 (see table 1), this corresponds to USD 1475 for Denmark, USD 5155 for Norway, and 1946 for Sweden.) However, in Finland the cost was found to be significantly lower for surgical treatment. The difference was FMK 7354 (USD 1599). Differences in outcome between the four countries could be explained to a large extent by differences in the cost of operation. The ratios between the cost of the operation per se and that of omeprazole in the medical treatment arm were 1.1, 1.7, and 1.2 in Denmark, Norway, and Sweden, but only 0.6 in Finland.

The cost of transportation was slightly higher in the medical treatment arm due to a larger number of visits but the cost of transportation was marginal in relation to the other cost estimates.

The indirect cost (that is, loss of production due to GORD related absence from work) was much higher in the surgical treatment arm in all countries (table 4). Taking indirect costs into account, the total cost per employed patient was higher in the surgical arm in all countries. The ratios between the total costs in the two treatment arms were 1.8 in Denmark, 1.1 in Finland, 2.1 in Norway, and 1.9 in Sweden.

SENSITIVITY ANALYSIS

Applying the two extreme cost scenarios, one favouring medical and the other surgical treatment, we found that the results were insensitive even to relatively large changes in unit costs. Even when all critical costs, except that of medication, were changed by as much as $20 \%$, the direct medical cost remained lower in the medical treatment arm in Denmark, Norway, and Sweden but higher in Finland.

However, when applying the scenario favouring surgery, the difference in direct medical cost ceased to be significant in Denmark and Sweden. Likewise, in the scenario favouring medical treatment, the difference between the therapeutic strategies became non-significant in Finland.

Table 4 Cost per patient during five years on therapy

\begin{tabular}{|c|c|c|c|c|c|c|c|c|}
\hline & \multicolumn{2}{|c|}{ Denmark (DKK) } & \multicolumn{2}{|c|}{ Finland (FMK) } & \multicolumn{2}{|c|}{ Norway (NOK) } & \multicolumn{2}{|c|}{ Sweden (SEK) } \\
\hline & Medical & Surgery & Medical & Surgery & Medical & Surgery & Medcial & Surgery \\
\hline Operation & 3463 & 34530 & 2307 & 23000 & 6619 & 66000 & 4188 & 41759 \\
\hline Endoscopy & 337 & 481 & 192 & 275 & 267 & 381 & 426 & 608 \\
\hline Visit to doctor & 2525 & 1258 & 1861 & 927 & 840 & 419 & 2445 & 1218 \\
\hline Visit to nurse & 290 & 280 & 147 & 142 & 96 & 93 & 369 & 357 \\
\hline Reoperation & 0 & 1112 & 0 & 644 & 0 & 2125 & 0 & 1594 \\
\hline Hernia & 117 & 2898 & 67 & 1667 & 107 & 2646 & 152 & 3753 \\
\hline Medication & 29946 & 4822 & 34872 & 5438 & 36665 & 5923 & 33728 & 5055 \\
\hline Direct medical cost & 36678 & 45381 & 39446 & 32092 & 44595 & 77587 & 41308 & 54344 \\
\hline Difference in direct medical cost & -8703 & & 7354 & & -32992 & & -13036 & \\
\hline $95 \% \mathrm{CI}$ for the difference & \multicolumn{2}{|c|}{$(-12178,-5229)$} & \multicolumn{2}{|c|}{$(4214,10493)$} & \multicolumn{2}{|c|}{$(-37668,-28317)$} & \multicolumn{2}{|c|}{$(-16786,-9286)$} \\
\hline Transportation & 361 & 279 & 341 & 263 & 540 & 416 & 489 & 377 \\
\hline Direct costs & 37039 & 45660 & 39787 & 32355 & 45135 & 78003 & 41797 & 54721 \\
\hline Indirect costs ${ }^{1}$ & 9890 & 39222 & 4147 & 16448 & 8313 & 32966 & 11902 & 47200 \\
\hline Total $^{1}$ & 46929 & 84882 & 43934 & 48803 & 53447 & 110969 & 53699 & 101921 \\
\hline
\end{tabular}

${ }^{1}$ Per patient employed at baseline. 
In a second sensitivity analysis, the cost for each patient lost to follow up was extrapolated to the end of the five year study period. This was found to increase the cost somewhat more in the medical treatment arm than in the surgical treatment arm. However, the direct medical cost remained significantly lower in the medical treatment arm in Denmark, Norway, and Sweden (differences were DKK 7437, NOK 31 179, and SEK 11 941, respectively), and significantly higher in Finland (FMK 9215).

Applying an additional assumption in the third sensitivity analysis - that is, an estimated consumption of omeprazole $20 \mathrm{mg}$ per day while waiting for surgery (on average 71 days) - increased the cost in the surgical treatment arm but had no effect on the main conclusions. Again, surgical treatment remained significantly less costly in Finland.

Finally, when we included the cost of protocol driven visits and endoscopies to the direct medical cost these increased somewhat more in the surgical arm but the main results remained the same in all countries.

\section{Discussion}

The present study, comparing monetary resource consumption by medical and surgical treatment of GORD, has the obvious advantage of applying a prospective protocol setting where costs were recorded when they occurred in the management of strictly comparable groups of patients. This methodological approach increases the precision of the cost estimates relevant to the two treatment options which is particularly true when our data are compared with the findings of earlier modelling studies.

Using a Markov model to assess the cost utility of long term omeprazole treatment versus laparoscopic fundoplication, Heudebert and colleagues ${ }^{8}$ reported an analysis which applied the perspective of a third party payer within the USA. Basically, both strategies were equally effective but omeprazole was found to be less expensive within the five year time horizon. Depending on the assumptions introduced into the model, a "break even" point between the two strategies was reached after about 10 years. Other studies, applying modelling and/or retrospective methodological approaches, have favoured surgical treatment reaching a "break even" point after a shorter time period of comparison. ${ }^{9}{ }^{12}$ Accordingly, most studies comparing the cost effectiveness of modern therapies for GORD have come to the conclusion that the surgical strategy is most cost effective in the long term perspective.

It is interesting to note that the present prospective, controlled, randomised study confirmed important observations in the earlier modelling and/or retrospective studies. Firstly, when interest was focused on direct medical costs, the relative costs of the operation and medication had a paramount impact on the final outcome. Other cost items were by and large marginalised. It follows from this basic observation that the result of a comparison between the costs of medical and surgical treatment of GORD will, by necessity, vary from one country to another and probably also from one time period to another. Among the four Nordic countries participating in the study, Finland had the lowest relative cost of an operation. Consequently, the five year direct cost of surgery was significantly higher than that of omeprazole in Denmark, Norway, and Sweden whereas the opposite was found in Finland.

Secondly, the study lends support to the hypothesis that the costs of medical treatment are relatively constant over time whereas those of surgical treatment are associated with a huge initial investment followed by low annual costs, eventually resulting in surgical treatment becoming less expensive than medical treatment with time.

Inclusion of indirect costs into health economic analyses is to some extent controversial but the results from this study indicate that ignoring loss of production due to absence from work would give rise to a considerable underestimation of the cost burden for the society inflicted by, for example, surgery. Furthermore, while in this study it was not possible to assess the cost of disease related decreases in productivity while at work, there is some evidence that this cost may be substantial for GORD. ${ }^{18}$

To comprehensively interpret the present observations and calculations we have to consider the limitations of the methodology presently applied. Even though our resource data base came from a randomised clinical trial, some uncertainty in the resulting estimates of costs is inevitable. A typical problem when utilising a clinical trial for collection of resource data is the inclusion/exclusion of protocol driven costs - that is, costs that are mandated by the trial protocol but which are unlikely to occur in clinical practice. In the present study we incorporated rules to exclude protocol driven visits and endoscopies from the cost analysis. Interestingly, we found that even when including those costs, the main outcomes remained unchanged. Another comment relevant to the protocol is that it defined the lowest dose of omeprazole $(20 \mathrm{mg})$ to be given as maintenance treatment. Today it is well known that some patients can be maintained on a lower dose. ${ }^{19}$

A general problem in health economic evaluations is the uncertainty inherent in cost estimates. A potential problem with this particular study is that the cost estimates may stem primarily from charges rather than estimates of the true costs. However, the sensitivity analysis revealed that even large changes in the cost estimates did not alter the sign of the differences found in direct medical costs.

There are also other methodological aspects which have to be taken into consideration. Pooling of data across countries regarding resource consumption in non-monetary terms can be questioned, especially pooling of time for travelling and number of days on sick leave. Furthermore, the patient completed the questionnaire on travelling and sick leave every six months. This rather long period may give rise to recall errors. Furthermore, the response rate 
decreased somewhat during the course of the study. Due to these limitations, estimates of direct medical costs were deemed to be more reliable than estimates of total costs. However, the present approach to obtain a total cost analysis is the most complete so far applied to GORD patients.

In general terms it is always questionable as to which time span should be applied to be most relevant on a strict comparison of these two treatment options. It is well known that individual decision makers sometimes take rather limited time horizons into account. From a societal point of view it may be more significant to cover a limited time period. The main reason for this is that the available health care technologies, and these associated costs, are prone to change. For instance, laparoscopic fundoplication has replaced open fundoplication in many hospitals. Laparoscopic ARS has been shown to be less costly than open surgery as the number of days in hospital and also the number of days on sick leave is reduced. ${ }^{20-26}$ In a recent Swedish prospective trial comparing the total costs of open and laparoscopic fundoplication, it was found that the total societal cost during the first postoperative year was reduced from SEK 74608 to SEK 40289 by applying the latter operative technique. ${ }^{20}$ On the other hand, the costs of PPIs are likely to fall substantially with the passage of time. Furthermore, extrapolation of costs and effectiveness is always inferior to prospective data collection.

In summary, this study provides useful information relevant to the long term management of patients with GORD. Direct medical costs over five years (costs of medication, surgery, visits, and examination) were found to be significantly lower for omeprazole treatment than for surgical treatment in Denmark, Norway, and Sweden. However, in Finland the direct medical cost was found to be significantly lower for ARS. Including indirect costs (loss of production due to GORD related sick leave), we found the costs of surgical treatment to increase substantially.

A three year interim analysis of this study was presented at the American Gastroenterological Association Meeting, Washing-
ton, DC, May 1997. The five year results were presented at the ton, DC, May 1997. The five year results were presented at the American Gastroenterological Association Meeting, San Diego, May 2000. The study was supported by the Swedish
Research Council and AstraZeneca, Mölndal, Sweden.

1 Howard PJ, Heading RC. Epidemiology of gastrooesophageal reflux disease. World F Surg 1992;16:288-93. 2 Lööf L, Götell P, Elfberg B. The incidence of reflux oesophagitis. Scand $\mathcal{F}$ Gastroenterol 1993;28:113-18.

3 Klinkenberg-Knol EC, Festen HP, Jansen JB, et al. Long-term treatment with omeprazole for refractory reflux oesophagitis: efficacy and safety. Ann Intern Med 1994;121 $161-7$
4 Hallerbäck B, Unge P, Carling L, et al. Omeprazole or ranitidine in long-term treatment of reflux oesophagitis. Gastroenterology 1994;107:1305-11.

5 Peracchia A, Bancewicz J, Bonavina L, et al. Fundoplication is an effective treatment for gastro-oesophageal reflux disease. Gastroenterol Int 1995;8:1-7.

6 Isolauri J, Luostarinen M, Viljakka M, et al. Long-term comparison of anti-reflux surgery vs conservative therapy for reflux oesophagitis. Ann Surg 1997;225:295-9.

7 Kahrilas PS. Laparoscopic antireflux surgery: silver bullet or the emperor's new clothes? Am f Gastroenterol 1999;7: 1721-3.

8 Heudebert GR, Marks R, Wilcox CM, et al. Choice of longterm strategy for the management of patients with severe oesophagitis: a cost-utility analysis. Gastroenterology 1997; 112:1078-86.

9 Coley CM, Barry MJ, Spechler SJ, et al. Initial medical vs surgical therapy for complicated or chronic gastroesophageal reflux disease (GORD): A cost-effectiveness analysis. Gastroenterology 1993;104:A5.

10 van den Boom G, Hameeteman W, Dallemagne B, et al. Cost effectiveness of medical versus surgical treatment in patients with severe or refractory gastroesophageal reflux disease in the Netherlands. Scand $\mathcal{f}$ Gastroenterol 1996;31: $1-9$.

11 Viljakka M, Nevalainen J, Isolauri J. Lifetime costs of surgical versus medical treatment of severe gastro-oesophageal reflux disease in Finland. Scand F Gastroenterol 1997;32: $776-2$.

12 Hurley S, Mckeage S, Ngaei G. Cost-effectiveness of primary medical therapy vs surgery for the treatment of gastroesophageal reflux disease (GORD). Aust $f$ Hosp Pharm 1996;26:171

13 Lundell L, Dalenbäck J, Hattlebakk J, et al. Outcome of open anti-reflux surgery as assessed in a Nordic multicentre prospective clinical trial. Eur F Surg 1998;164:751-7.

14 Sandmark S, Carlsson R, Fausa O, et al. Omeprazole or ranitidine in the treatment of reflux oesophagitis. Scand 7 Gastroenterol 1988;23:625-32.

15 Lundell L, Dalenbäck J, Janatuinen E, et al. Comprehensive 1 -year costs analysis of open anti-reflux surgery in Nordic countries. Br f Surg 1998;85:1002-5.

16 Lundell L, Miettinen P, Myrvold HE, et al. Long term management of gastro-oesophageal reflux disease with omeprazole or open antireflux surgery. Eur 7 Gastroenterol Hepatol 2000;12:879-87.

17 Henke CJ, Levin TR, Henning JM, et al. Work loss costs due to peptic ulcer disease and gastroesophageal reflux disease in a health maintenance organisation. Am $\mathcal{f}$ Gastroenterol 2000;95:788-92.

18 Lundell L, Miettinen P, Myrvold H, et al. Continued (5 year) follow-up of a randomised clinical study comparing antireflux surgery and omeprazole in gastro-oesophageal reflux disease. F Am Coll Surg 2001;192:172-9.

19 Carlsson R, Galmiche JP, Dent J, et al. Prognostic factors influencing relapse of oesophagitis during maintenance therapy with antisecretory drugs: a meta-analysis of long-term omeprazole trials. Aliment Pharmacol Ther 1997; 11:473-82.

20 Blomqvist AMK, Lönroth H, Dalenbäck J, et al. Laparoscopic or open fundoplication? A complete cost analysis. Surg Endosc 1998;12:1209-12.

21 Perdikis G, Hinder RH, Lund RJ, et al. Laparoscopic Nissen fundoplication: Where do we stand? Surg Laparosc Endosc 1997;1:17-21.

22 Carbone R, Peters JH, Heimbucher S, et al. A contemporaneous comparison of hospital charges for laparoscopic and open Nissen fundoplication. Surg Endosc 1995;9:151-5.

23 Laycock WS, Oddsdottir M, Franco A, et al. Laparoscopic Nissen fundoplication is less expensive than open Belsey Mark IV. Surg Endosc 1995;9:426-9.

24 Rattner DW, Brooks DC. Patient satisfaction following laparoscopic and open antireflux surgery. Arch Surg 1995;130:289-93.

25 Richards KF, Fisher KS, Flores JH, et al. Laparoscopic Nissen fundoplication: cost, morbidity, and outcome compared with open surgery. Surg Laparosc Endosc 1996;6:1403 .

26 Heikkinen TJ, Haukipuro K, Koivukangas $\mathrm{P}$, et al. Comparison of costs between laparoscopic and Nissen fundoplication: a prospective randomized study with a 3-month followup. $\mathcal{F}$ Am Coll Surg 1999;188:368-76. 